\title{
EL DERECHO REAL DE CONSERVACIÓN: UN MECANISMO DE PAGO POR SERVICIOS AMBIENTALES
}

\author{
CONSERVATION EASEMENTS: A PAYMENT FOR \\ ENVIRONMENTAL SERVICE
}

\begin{tabular}{|c|c|}
\hline \multicolumn{2}{|c|}{ ARTÍCULO INÉDITO DE INVESTIGACIÓN } \\
\hline CÓMO CITAR ESTE ARTÍCULO (CHICAGO) & $\begin{array}{l}\text { García García, Sara. "El derecho real de } \\
\text { conservación: Un mecanismo de pago } \\
\text { por servicios ambientales". Revista de } \\
\text { Derecho Aplicado LLM UC } 6 \text { (2020). } \\
\text { doi: 10.7764/rda.o.6.17987. }\end{array}$ \\
\hline REVISTA DE DERECHO APLICADO LLM UC & $\begin{array}{l}\text { Número } 6 \\
\text { Diciembre } 2020 \\
\text { ISSN: } 245^{2-4344}\end{array}$ \\
\hline & $\begin{array}{l}\text { Recepción: } 22 \text { de julio, } 2020 \\
\text { Aceptación: } 28 \text { de agosto, } 2020\end{array}$ \\
\hline
\end{tabular}




\section{Resumen}

La figura de la conservation easement ha surgido en el ámbito anglosajón como un mecanismo jurídico nuevo y efectivo de conservación de la naturaleza basado en servicios ambientales. En este trabajo se analiza el derecho real de conservación, su versión latinoamericana, debido al interés que está despertando y a la eficacia en la protección del medio ambiente que, según parecen demostrar experiencias concretas, proporciona esta figura. El objetivo es analizar si en verdad estamos ante un nuevo derecho real, basado además en el moderno concepto de los servicios ambientales, o si materialmente su naturaleza es otra y, por tanto, requiere un tratamiento jurídico adaptado.

Palabras clave: Medio ambiente, servidumbres, servicios, recursos naturales, conservación

\section{Abstract}

Conservation easement has emerged in the Anglo-Saxon world as a new and effective legal mechanism for nature conservation based on environmental services. In this paper we analyze the derecho real de conservación, its Latin American version. This derecho real is having a great development as an effective technique for environmental protection, according to concrete experiences. The objective is to analyze if we are really facing a new derecho real, based on the modern concept of environmental services, or if, on the contrary, it is another kind of technique which requires another type of reglamentation.

Keywords: Environment, easements, services, natural resources, conservation 


\section{Sara García García}

Universidad de Valladolid

Valladolid, España

sara.garciag@uva.es

Universidad de Valladolid

Valladolid, Spain

sara.garciag@uva.es
Sara García García es profesora asociada de Derecho en la Universidad de Valladolid, España, perteneciente al área de Derecho Administrativo. Investigadora predoctoral en derecho administrativo, especializada en medioambiente.

Sara García García is Associate Professor of Law at University of Valladolid, Spain. Administrative Law Area. PhD researcher in Administrative Law, specialized in Environmental Law. 


\section{INTRODUCCIÓN A LAS CONSERVATION EASEMENTS O SERVIDUMBRES DE CONSERVACIÓN}

La figura de la conservation easement ha surgido en el ámbito anglosajón como un mecanismo jurídico nuevo y efectivo de conservación de la naturaleza basado en servicios ambientales. Allí son definidas como un acuerdo legal y voluntario entre el propietario de una finca y un tercero, persona física o jurídica o el Estado, con el fin de restringir el uso de sus recursos naturales, incluso de forma permanente ${ }^{1}$. Se trata de un acuerdo de limitación del dominio para la protección de los valores naturales de un espacio²; una limitación establecida ob rem para el uso o gestión de un terreno basada en objetivos conservacionistas, ligada a la propiedad y no al propietario y que, por tanto, pasaría a imponerse a los sucesivos titulares ${ }^{3}$. El gravamen recaído sobre el espacio sirviente que propone esta figura podrá establecerse bien a favor del espacio dominante, para la conservación de sus valores o servicios ambientales - lo que sería una servidumbre ecológica predial o, en el ámbito anglosajón, la denominada easement tipo appurtenant-; o bien a favor del propio dueño de la finca, que restringe las actividades de uso de la tierra para proteger sus valores naturales - lo que vendría a tener un carácter de servidumbre más personal, denominada in-gross - ${ }^{4}$. Esta restricción de las facultades del dueño —al estudiar en profundidad la figura - se entiende sobre la base de una regulación pública previa cuyo cumplimiento se pretende.

Pese a su origen, es en los países latinoamericanos donde, bajo la denominación de servidumbre de conservación o también derecho real de conservación, esta figura está expe-

1 Adena Rissman y otros, "Conservation Easements: Biodiversity Protection and Private use", Conservation Biology 2I, n. ${ }^{\circ}$ (2006): 710: "Las servidumbres de conservación son un acuerdo voluntario entre propietarios privados de tierras o entre un propietario y el poder público cuyo objetivo es limitar el uso de la tierra, teniendo como fin conservar recursos naturales incluso de forma permanente".

2 Elizabeth Byers y Karin Marchetti Ponte, The Conservation Easement Handbook (Washington, D. C.: Land Trust Alliance, 2005), 7 .

3 Colin T. Reid, "The Privatisation of Biodiversity? Possible New Approaches to Nature Conservation in the UK", Journal of Environmental Law 23 (20II): 2II.

4 Ignacio Carvajal Gómez, "El derecho real de conservación", Revista de Derecho Ambiental 2 (mayo 2010): I86. 
rimentando mayor desarrollo como instrumento para la protección expresa y exclusiva de servicios ambientales ${ }^{5}$.

En la mayoría de los ordenamientos que están asumiendo como nueva técnica estas easements (destacan Puerto Rico, Chile, Ecuador, Costa Rica y Perú) ${ }^{6}$ se presentan como un contrato voluntario a través del cual se comprometen limitaciones para fomentar o conservar servicios ambientales; limitaciones que serán compensadas económicamente ${ }^{7}$. Esto se traduce en un contrato, celebrado entre particulares o con una entidad pública, en el que se acuerda la restricción del uso que puede hacerse de un terreno en pro de la conservación de los recursos y servicios ambientales. Esa limitación es compensada con fondos por lo general públicos y entregados en forma directa a los agricultores, dueños del terreno.

5 Hay autores que pretenden marcar distancias y aportan un punto de distinción conceptual entre las conservation easements anglosajonas, entendidas como el "acuerdo legal por parte voluntaria del dueño que limita el uso de una propiedad con el fin de conservar los recursos naturales", y su interpretación bajo la figura de las servidumbres de conservación, que definen como la "concesión para utilizar terrenos que le da el derecho a una persona para hacer uso de la tierra que es propiedad de otra persona, o para restringir el uso de la tierra que se somete a la servidumbre” (Carvajal Gómez, “El derecho...”, I85), lo cierto es que en la mayoría de los casos (Ecuador, Costa Rica o Perú) se habla de las servidumbres de conservación como "el acuerdo voluntario de dos propietarios para imponer gravámenes o limitaciones de uso sobre una parte o la totalidad de su predio, con el propósito de garantizar la provisión de un servicio ambiental o ecológico en beneficio de otro predio, y con ello contribuir a la conservación, protección, restauración, mejoramiento y manejo adecuado de los recursos naturales y de los valores ambientales existentes en este"; por lo tanto, son dos versiones muy similares de una misma figura: José Luis Capella, Miriam Cerdán y Pedro Solano, Manual de instrumentos legales para la conservación privada en el Perú (Lima: Sociedad Peruana de Derecho Ambiental, 2007), 3.

Destacan en este punto Puerto Rico, con la Ley r83 de Servidumbre de Conservación, del 27 de diciembre de 200I, que se define como "derecho real consistente en la facultad de conservar el patrimonio ambiental de un predio o de ciertos atributos o funciones de este. Este derecho se constituye en forma libre y voluntaria por el propietario del predio en beneficio de una persona natural o jurídica determinada”; y Chile, con la Ley 20.930 , del 26 de junio de 2016 , que establece el derecho real de conservación medioambiental, que se trata como un "derecho real consistente en la facultad de conservar el patrimonio ambiental de un predio o de ciertos atributos o funciones de este. Este derecho se constituye en forma libre y voluntaria por el propietario del predio en beneficio de una persona natural o jurídica determinada”. De esta forma, no se trataría de un derecho real atípico, porque viene establecido por la Ley 20.930 de $20 \mathrm{r} 6$.

7 "Acuerdo voluntario de dos propietarios para imponer gravámenes o limitaciones de uso sobre una parte o la totalidad de su predio, con el propósito de garantizar la provisión de un servicio ambiental o ecológico en beneficio de otro predio, y con ello contribuir a la conservación, protección, restauración, mejoramiento y manejo adecuado de los recursos naturales y de los valores ambientales existentes en este”. Capella, Cerdán y Solano, Manual..., 3r. 


\section{CONTEXTO DE LA FIGURA: DEL TRADICIONAL DERECHO REAL DE SERVIDUMBRE AL NUEVO CONCEPTO DE SERVICIO AMBIENTAL}

\section{I. Breve repaso de la servidumbre}

La servidumbre es un derecho real. En el Código Civil español la figura de la servidumbre queda recogida en el artículo 530 como "gravamen impuesto sobre un inmueble en beneficio de otro perteneciente a distinto dueño" (servidumbre predial); el beneficio puede imponerse en favor de una persona o comunidad, estando entonces ante servidumbres personales (artículo 531). Doctrinalmente, la servidumbre se describe como un "derecho real limitativo del dominio que atribuye a su titular el goce o aprovechamiento de un inmueble ajeno, goce que restringe o limita a sólo determinados usos, servicios o utilidades" ${ }^{8}$. La servidumbre otorga entonces ciertas capacidades a su titular sobre bienes que son ajenos, que podrán consistir en una conducta y un uso activo (servidumbres positivas) o en la exigencia de una abstención por parte de un tercero (servidumbres negativas) ${ }^{9}$.

Existe una tercera tipología de servidumbres, cuyo objeto consiste en atender directa y principalmente al interés colectivo, teniendo como derecho aplicable el administrativo. De manera usual, estas servidumbres se aplican en ámbitos concretos como la agricultura, aguas, minas, montes, telecomunicaciones, etcétera, y son susceptibles de ser englobadas bajo la denominación de servidumbres administrativas ${ }^{10}$. La servidumbre administrativa es, por lo general, utilizada más como una técnica de protección de bienes pertenecientes al dominio público que como un modo de establecimiento de un derecho de disfrute o beneficio sobre bienes ajenos ${ }^{11}$.

La relación entre la servidumbre y los recursos naturales o servicios ambientales no es nueva: existen servidumbres tradicionales que recaen sobre lo que hoy identificamos como servicios ambientales. Tal sería el caso de ciertas servidumbres de aguas (artículos 552 a 563 del Código Civil), de pastos (artículos 600 a 604) y leñas o de vistas (artículos 580 a 585).

8 Bernardo Gómez Corraliza, "El derecho real de servidumbre. Concepto. Naturaleza. Diferencias con figuras afines. Caracteres”, en Tratado de servidumbres, dir. por María Linacero de la Fuente (Madrid: Tirant lo Blanch, 2017), 39 .

9 Cfr. Mariano Alonso Pérez, "Reflexiones críticas en torno a la servidumbre en el Código Civil", en Historia de la propiedad: servidumbres y limitaciones del dominio, coord. por Salustiano de Dios de Dios, Javier Infante, Ricardo robledo y Eugenia Torijano (Madrid: Servicio de Estudios del Colegio de Registradores, 2009), 8I7.

10 Cfr. Ángel Luis Rebolledo Varela (director), Tratado de servidumbres (Navarra: Thomson Aranzadi, 2007), 87-88.

11 Cfr. Roberto Parejo Gamir y José María Rodríguez Oliver, Lecciones de dominio público (Madrid: ICAI, I976), II5 y ss. 


\subsection{El concepto de servicio ambiental}

El servicio ambiental se define, desde un punto de vista normativo, como "las funciones que desempeña un recurso natural en beneficio de otro recurso o del público" ${ }^{12}$. Sobre esa base, se califica un daño medioambiental como "el cambio adverso y mensurable de un recurso natural o el perjuicio de un servicio de recursos naturales, tanto si se produce directa como indirectamente" (artículo 2, apartado 2). De este modo, la figura del servicio ambiental se reconoce como una realidad vulnerable y que debe ser protegida; un criterio jurídico que debe integrarse ya en las normas más básicas de protección del medio ambiente, ligado por completo a la figura de los recursos naturales.

El servicio ambiental es, por tanto, un beneficio, una utilidad que obtenemos de la naturaleza y que interesa proteger. Esa utilidad es un posible vínculo que podríamos encontrar entre el servicio ambiental y una servidumbre: la utilitas, que puede consistir en cualquier ventaja o beneficio que el bien pueda otorgar al titular del derecho sería la nota esencial caracterizadora de un derecho real de servidumbre ${ }^{13}$. La cuestión está en que el fin esencial del denominado derecho real de conservación o servidumbre de conservación no es otorgar una utilidad al titular de la servidumbre, sino fomentar la conservación de servicios ambientales a través del establecimiento de una limitación en un terreno y compensar dicha restricción.

\section{LOS PAgOS POR SERVICIOS AMBIENTALES}

Los pagos por servicios son nuevos mecanismos de orientación o fomento de la conservación de la naturaleza, basados en los servicios ambientales. Los diferentes sistemas de pago por servicios ambientales se han construido aplicando mecanismos de mercado, de tal forma que, a través de incentivos económicos, se pretende estimular al propietario o gestor de una tierra a introducir en sus planes el cuidado de los valores naturales. Los pagos por servicios se presentan, en definitiva, como una transferencia de recursos entre actores sociales con el objetivo de crear incentivos que fomenten un uso de la tierra ambientalmente adecuado $^{14}$.

La práctica de estos mecanismos cuenta ya con cierta experiencia. Costa Rica y Ecuador son dos de los países latinoamericanos más referenciados en los estudios sobre pagos por servicios ambientales. Costa Rica fue, de hecho, la primera nación en implementar un programa a largo plazo de pagos por servicios ambientales; y lo hizo desarrollando la

12 Artículo 2 de la Directiva sobre Responsabilidad Medioambiental 200//35 y su Ley española de transposición $26 / 2007$.

13 Cfr. Rebolledo Varela, Tratado..., 70-73.

14 Ángel Moreno Inocencio, "Pagos por servicios ambientales: Propuestas financieras y tributarias para su implantación en España”, Crónica Tributaria I63 (20I7): I49. 
figura a través de contratos renovables de carácter plurianual, auspiciados por un fondo de compensación financiera creado ad hoc por el Gobierno. De esta manera, el Estado costarricense proporciona una compensación financiera a los propietarios de bosques y plantaciones forestales por los servicios ambientales que prestan sus tierras, con lo que contribuyen en forma directa a la protección y mejora del medio ambiente. Crea así incentivos sólidos para que los propietarios de tierras cambien su comportamiento a uno ambientalmente adecuado ${ }^{15}$. Otro ejemplo de pago por servicios institucionalizado lo encontramos en el modelo actual desarrollado por la Política Agrícola Común de la Unión Europea (PAC): la ecocondicionalidad de sus pagos o el conocido como pago verde (greening), a través del cual se "remunera a los agricultores por realizar prácticas beneficiosas para el clima y el medio ambiente", constituyen auténticas compensaciones por conservar los servicios de la naturaleza ${ }^{16}$; son, "en toda regla, un mecanismo de pago por servicios ambientales", quedando sujetos al sistema legal establecido para estos ${ }^{17}$.

A través de estos mecanismos de pagos por servicios ambientales se retribuye a quienes, desarrollando una actividad económica, están garantizando la conservación y restauración de los ecosistemas, incentivándoles para que conserven la biodiversidad a la vez que hacen la correspondiente actividad económica; en palabras de Rodríguez-Chaves Mimbrero, con los pagos por servicios

se pretende conformar un marco contractual mediante el cual los titulares de las explotaciones agrarias asuman desarrollar un modelo de actividad agraria que genere externalidades positivas en los ámbitos mencionados, y por el cual, en apreciación del interés público de dichas externalidades, las administraciones públicas competentes las compensan, incentivan y retribuyen, como forma de reconocimiento por la sociedad de los servicios y prestaciones de carácter público que generan las explotaciones agrarias más allá de la retribución derivada de la venta en el mercado de sus productos ${ }^{18}$.

15 Rodrigo A. Arriagada, Erin O. Sills, Subhrendu K. Pattanayak y Paul J. Ferraro, "Combining Qualitative and Quantitative Methods to Evaluate Participation in Costa Rica's Program of Payments for Environmental Services", Journal of Sustainable Forestry 28, n. $^{\circ}{ }^{-5}$ (2009): $344^{-}$ $348,3^{6 r}$.

16 Pilar Martínez, Javier Castaño y María Blanco, "Simulador PAC: Lecciones del análisis del pago verde", Revista Española de Estudios Agrosociales y Pesqueros 248 (20I7): 15-37.

17 José María Rábade Blanco (coordinador), “GT-ı6. Pago por servicios ambientales: la PAC”, documento de trabajo (Madrid: Conama, 20I2), 7 y ss.

18 Blanca Rodríguez-Chaves Mimbrero, "Pago por servicios ambientales (PSA) en el derecho europeo y en el derecho interno español: Apuntes sobre su situación actual y perspectivas”, $R e-$ vista Aranzadi de Derecho Ambiental 24 (20I3): 98. 


\section{EL DERECHO REAL DE CONSERVACIÓN: UN MECANISMO MÁS DE PAgO POR SERVICIOS AMBIENTALES}

Expuesto lo anterior, resta por determinar la verdadera naturaleza de este denominado derecho real de conservación.

En Chile se está desarrollando una corriente que pretende dibujar este derecho real de conservación como una figura semejante a los derechos reales de aprovechamiento parcial previstos en algunas regulaciones autonómicas españolas, como la aragonesa o la catalana. Tal comparación es errónea, pues el presupuesto del que parten estas figuras autonómicas - una explotación o aprovechamiento forestal que se compensaría, en cualquier caso, con una conservación del resto de elementos naturales del terreno- nada tiene que ver con la definición que se ofrece de la servidumbre de conservación: estos derechos de aprovechamiento parcial serían próximos a una servidumbre personal, cuyo objetivo es la obtención de una utilidad o aprovechamiento para su titular a cambio, en este caso, de rehacer y conservar los recursos utilizados. Sin embargo, la servidumbre de conservación parece diseñarse como un mecanismo destinado a la conservación de servicios ambientales, ejecutado a través de acuerdos de limitación de actividades sobre la base, por lo general, del cumplimiento de un deber público.

Como se expone, esta nueva servidumbre es un instrumento de fomento de la conservación de la naturaleza articulado a través de convenios de colaboración con particulares y entidades públicas y privadas. Así descrita, tiene un contenido prácticamente equivalente a instrumentos de pago por servicios como el denominado contrato territorial en España.

La figura del contrato territorial, desarrollada por el Real Decreto 1336/2011, del 3 de octubre, es un instrumento de política económica general enfocado al medio rural español, que utiliza la Administración Pública como medio de impulso para que la actividad agraria apueste por un enfoque integral y multifuncional en beneficio de un desarrollo sostenible y de la conservación adecuada de la naturaleza ${ }^{19}$. Se presenta así como un contrato de suscripción voluntaria, tanto para las comunidades autónomas que opten por ponerlos en práctica, como para los titulares de las explotaciones agrarias, cuyo objeto principal consiste en conservar la naturaleza (artículo 1).

Son evidentes las similitudes que esta figura tradicional en el ámbito europeo comparte con la nueva servidumbre de conservación americana: ambos son contratos que utilizan el poder público como incentivo para la conservación de la naturaleza. Ambos son medios, formalmente distintos, para compensar por la conservación de servicios ambientales.

19 Cfr. Preámbulo del Real Decreto I336/20II, del 3 de octubre, por el que se regula el contrato territorial como instrumento para promover el desarrollo sostenible del medio rural. 


\section{CONCLUSIONES}

El derecho real de conservación o servidumbre de conservación se presenta como un contrato voluntario a través del cual se fomenta la conservación por particulares de servicios ambientales mediante limitaciones de su actividad o en el uso de un terreno; limitaciones que serán compensadas económicamente por el poder público.

Pese a la denominación que se quiera dar a esta nueva figura, la realidad es que su contenido concreto nos obliga a desvincularla por completo del ámbito de los derechos reales e insertarla en el de los pagos por servicios ambientales: estas servidumbres son mecanismos de conservación de servicios ambientales de carácter voluntario que responden a medidas de impulso u obligaciones de conservación impuestas por el poder público. Este cuidado de la naturaleza resulta compensado con una contraprestación económica por el poder público, en esencia, sin perjuicio de que pueda acordarse inter privatos con el fin, en general, de cumplir con el deber público de conservar el entorno. 


\section{BIBLIOGRAFÍA}

- Alonso Pérez, Mariano. "Reflexiones críticas en torno a la servidumbre en el Código Civil”. En Historia de la propiedad: Servidumbres y limitaciones del dominio, coordinado por Salustiano de Dios de Dios, Javier Infante, Ricardo robledo y Eugenia Torijano. Madrid: Servicio de Estudios del Colegio de Registradores, 2009.

- Arriagada, Rodrigo A., Erin O. Sills, Subhrendu K. Pattanayak y Paul J. Ferraro. "Combining Qualitative and Quantitative Methods to Evaluate Participation in Costa Rica's Program of Payments for Environmental Services". Journal of Sustainable Forestry 28, n. ${ }^{\circ}$ 3-5 (2009): 343-367. doi: 10.1080/10549810802701192.

- Byers, Elizabeth y Karin Marchetti Ponte. The Conservation Easement Handbook. Washington, D. C.: Land Trust Alliance 2005.

- Capella, José Luis, Miriam Cerdán y Pedro Solano. Manual de instrumentos legales para la conservación privada en el Perú. Lima: Sociedad Peruana de Derecho Ambiental, 2007.

- Carvajal Gómez, Ignacio. "El derecho real de conservación”. Revista de Derecho Ambiental 2 (mayo 2010): 177-221.

- Gómez Corraliza, Bernardo. "El derecho real de servidumbre. Concepto. Naturaleza. Diferencias con figuras afines. Caracteres”. En Tratado de servidumbres, dirigido por María Linacero de la Fuente. Madrid: Tirant lo Blanch, 2017.

- Martínez, Pilar, Javier Castaño y María Blanco. "Simulador PAC: Lecciones del análisis del pago verde". Revista Española de Estudios Agrosociales y Pesqueros 248 (2017): 15-37.

- Moreno Inocencio, Ángel. “Pagos por servicios ambientales: Propuestas financieras y tributarias para su implantación en España”. Crónica Tributaria 163 (2017): 147-168.

- Parejo Gamir, Roberto y José María Rodríguez Oliver. Lecciones de dominio público. Madrid: ICAI, 1976.

- Rábade Blanco, José María (coordinador). "GT-16. Pago por servicios ambientales: La PAC”. Documento de trabajo. Madrid: Conama, 2012.

- Rebolledo Varela, Ángel Luis (director). Tratado de servidumbres. Navarra: Thomson Aranzadi, 2007. 
- Reid, Colin T. "The Privatisation of Biodiversity? Possible New Approaches to Nature Conservation Law in the UK". Journal of Environmental Law 23 (2011): 203-231. doi: 10.1093/jel/eqr005.

- Rissman, Adena, Lynn Lozier, Tosha Comendant, Peter Kareiva, Joseph M. Kiesecker, M. Rebecca Shaw y Adina M. Merenlender. "Conservation Easements: Biodiversity Protection and Private use". Conservation Biology 21, n. ${ }^{o} 3$ (2006): 709-718. doi: 10.1111/j.15231739.2007.00660.x.

- Rodríguez-Chaves Mimbrero, Blanca. "Pago por servicios ambientales (PSA) en el derecho europeo y en el derecho interno español: Apuntes sobre su situación actual y perspectivas". Revista Aranzadi de Derecho Ambiental 24 (2013): 81-128. 